\title{
EL SITIO ALERO PICTON 1: \\ NUEVO REGISTRO DE ARTE RUPESTRE PARA LOS CANALES FUEGUINOS
}

CAMILA MUÑOZ S. ${ }^{a}$, ROSARIO CORDERO F.a \& DIEGO ARTIGAS S. C. ${ }^{b}$

\begin{abstract}
RESUMEN
La siguiente nota tiene como objetivo informar acerca de la existencia de un sitio emplazado en isla Picton, canal Beagle, provincia de Antártica Chilena, XII región de Magallanes -Chile-, que presenta manifestaciones de arte rupestre asociadas a lentes de conchal de carácter arqueológico. A partir de una breve descripción de los dos motivos identificados, se enmarca el hallazgo dentro de los estudios existentes de arte rupestre en los canales australes, para de esta forma realizar una aproximación inicial al tipo de arte rupestre que desarrollaron los grupos canoeros en la zona.
\end{abstract}

PALABRAS CLAVE: arte rupestre, isla Picton, canales australes.

PICTON 1 ROCKSHELTER SITE:

NEW RECORD OF ROCK ART FOR THE FUEGUIAN CHANNELS

\begin{abstract}
The goal of this note is to inform about the existence of a rock art site associated to an archaeological shell midden located at Picton island, Beagle channel, province of Chilean Antarctica, XII region of Magallanes - Chile. A brief description of two identified motifs in addition to the existing studies of rock art in the southern channels, allows an initial approach to the type of rock art developed by the marine hunter gatherers groups in this area.

KEY WORDS: rock art, Picton island, southern channels.

\section{INTRODUCCIÓN}

La presente nota tiene como objetivo entregar los primeros antecedentes en relación

al sitio Alero Picton 1, yacimiento con

representaciones rupestres emplazado en la isla homónima, región de Magallanes y Antártica Chilena, Chile (Fig. 1). Este sitio, junto con otros identificados (Fage, 2006; Mena et al. 2011; González et al. 2014), pasa a ser parte del

a Consejo de Monumentos Nacionales, Viña del Mar 20, Providencia, Santiago-Chile, camila.munoz.soto@gmail.com, rosariocorderof@gmail.com

b Universidad SEK, Fernando Manterola 0789, Providencia, Santiago-Chile, diego.artigas@usek.cl
\end{abstract}



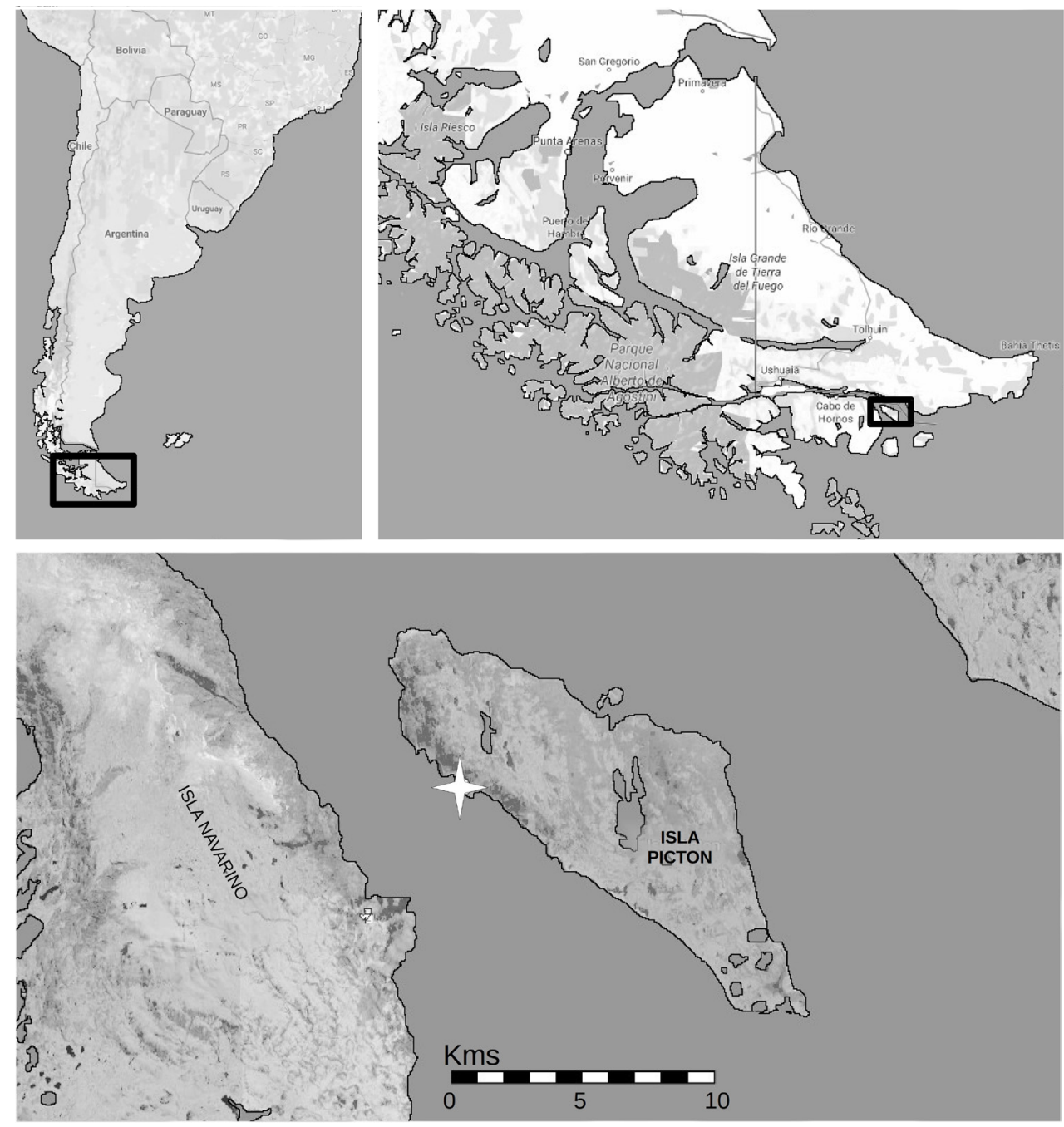

Fig. 1. Ubicación del sitio Alero Picton 1.

escaso registro de este tipo de evidencias para los canales patagónicos del extremo austral de Chile y del archipiélago fueguino.

La identificación del sitio se dio en el marco de una visita realizada por personal del Consejo de Monumentos Nacionales de Chile a isla Navarino que, al contar con el apoyo logístico de la Armada de Chile, permitió visitar el sector suroeste de Isla Picton, área en donde vivió la última familia que habitó la isla hasta aproximadamente el año 1987. En dicha visita se contó con la presencia del Sr. Jorge Quelín Nancul, uno de los últimos habitantes de este lugar, quien mencionó la presencia de una pared con pinturas realizadas por "los indios" que habitaron antiguamente el sector.

A partir de lo anterior, se dio paso a una prospección dirigida en la que se logró identificar dos grandes conchales: el primero asociado a los restos de la casa habitada antiguamente por la familia Quelín-Nancul, el que se encontraba altamente disturbado y sin material arqueológico asociado; mientras que el segundo fue identificado en un perfil expuesto a $18,5 \mathrm{~m}$ del sitio y asociado a manifestaciones rupestres, en donde se registró material lítico compuesto en su mayoría por lascas de basalto. Asimismo, se observaron varios lentes de conchal en sectores erosionados que podrían formar parte de un mismo yacimiento. 


\section{EL SITIO ALERO PICTON 1}

El sitio denominado como Alero Picton 1 se encuentra emplazado en el sector suroeste de la isla homónima, específicamente en la tercera terraza a $12 \mathrm{msnm}$, la cual presenta exposición hacia el suroeste. Este corresponde a un reparo rocoso de $42 \mathrm{~m}$ de largo (Fig. 2), asociado a lentes de conchal compuestos por fragmentos de gastrópodos $\mathrm{y}$ restos óseos de animal, posiblemente aves, los que se extienden desde los pies del sitio hasta aproximadamente $18,5 \mathrm{~m}$ a su alrededor, tanto en la segunda (7-9 msnm) como tercera terraza (10$13 \mathrm{msnm}$ ), los cuales sólo se pueden apreciar en aquellos sectores expuestos y erosionados, tanto por la acción de animales como por la presencia de construcciones (galpón de esquila) ocupadas por antiguas familias que habitaron la isla.

A partir de la inspección de los conchales asociados, se logró registrar material lítico en superficie el cual fue georreferenciado y fotografiado. En su mayoría corresponden a desechos de talla de basalto (rocas bases), identificándose escasas lascas con retoque marginal, algunas utilizadas probablemente como raspador $y / 0$ raedera. Es importante recalcar que actualmente no vive gente en este sector de la isla, lo que ha permitido que el registro arqueológico se haya visto escasamente impactado en el último tiempo, siendo el principal agente de deterioro las manadas de caballos salvajes (baguales) que habitan el sector.

En cuanto a los motivos rupestres identificados en el sitio (Fig. 2), sólo se registró un total de dos representaciones, enmarcadas dentro del grupo definido como geométricos o esquemáticos (Gradín et al. 1976; Gradín, 1978), ambas plasmadas en el sector este del alero.

El primer motivo fue registrado, inicialmente, como una línea vertical principal en color rojo con cuatros trazados lineales que se desprenden diagonalmente de ésta, de $14 \mathrm{~cm}$ de ancho y 17 $\mathrm{cm}$ de largo, emplazada a 1,25 $\mathrm{m}$ del suelo actual del alero (Fig. 3).

El segundo motivo está compuesto por una línea paralela roja, de la que se desprenden cinco líneas verticales paralelas, de $18 \mathrm{~cm}$ de ancho y 28 $\mathrm{cm}$ de largo, a 1,42 $\mathrm{m}$ del suelo actual del sitio (Fig. 4). En el mismo se advierte la posible presencia

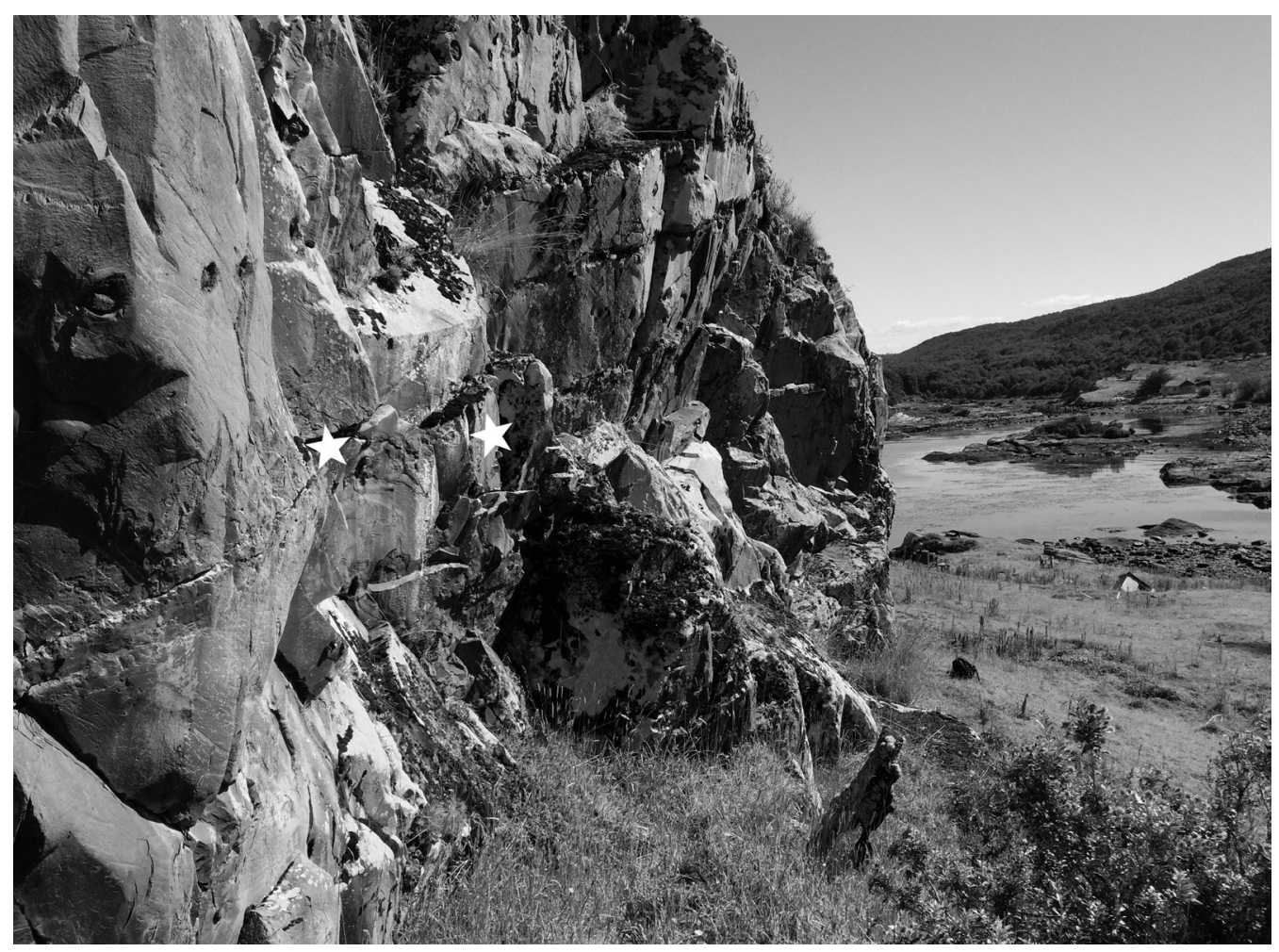

Fig. 2. Ubicación de las representaciones en el sector este del sitio. 

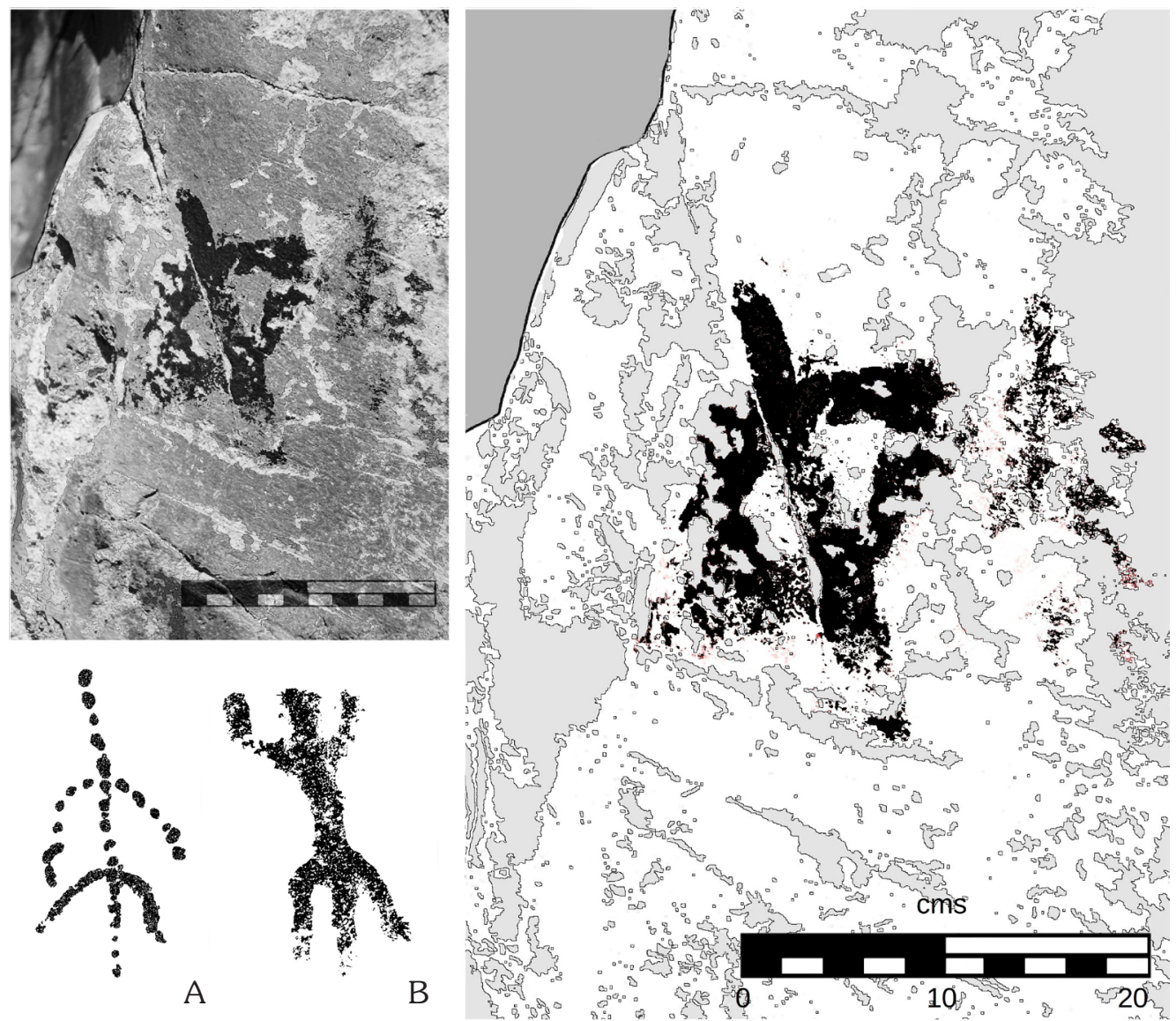

B

Fig. 3: Motivo 1 definido como antropomorfo en base a comparación con representación A) de Isla Madre de Dios (Fage, 2006) y motivo B) de Alero Martín González Calderón (González et al. 2014).

de estarcido anaranjado en la parte inferior de la representación.

Es necesario enfatizar que los motivos se encuentran en regular estado de conservación, observándose en el motivo 1 un alto grado de deplacamiento en torno a la representación, principalmente en ambos costados, mientras que en el motivo 2 se aprecian veladuras sobre toda la representación y deplacados en el área inferior izquierda.

En cuanto al resto del sitio, pese a que se recorrió toda su extensión, no se identificaron otros motivos. Sin embargo, se registraron manchas rojas en diferentes sectores, que luego de ser analizadas in situ y posteriormente a través del programa computacional DStretch (Harman, 2006), permitió llegar a la conclusión de que corresponden a vetas naturales de la roca y/o procesos de oxidación.

\section{COMPARACIONES CON OTRAS PINTURAS RUPESTRES DE PATAGONIA}

$\mathrm{Al}$ considerar los registros de arte rupestres presentes para Patagonia, tanto en el sector continental como en los canales, y al comprarlos con los motivos registrados en Alero Picton 1, se desprenden observaciones que permiten esbozar un panorama inicial del arte rupestre de los canales australes.

En el caso del territorio de Patagonia continental se han identificado una serie de sitios con arte rupestre a lo largo de los años (Bate, 1970, 1971; Lucero \& Mena, 2000; Carden, 2009; Gallardo, 2009; Sepúlveda, 2011; Artigas \& Muñoz, 2015; Muñoz \& Artigas, 2016, entre otros), lo que en un inicio permitió definir una serie de representaciones que se podían adscribir 


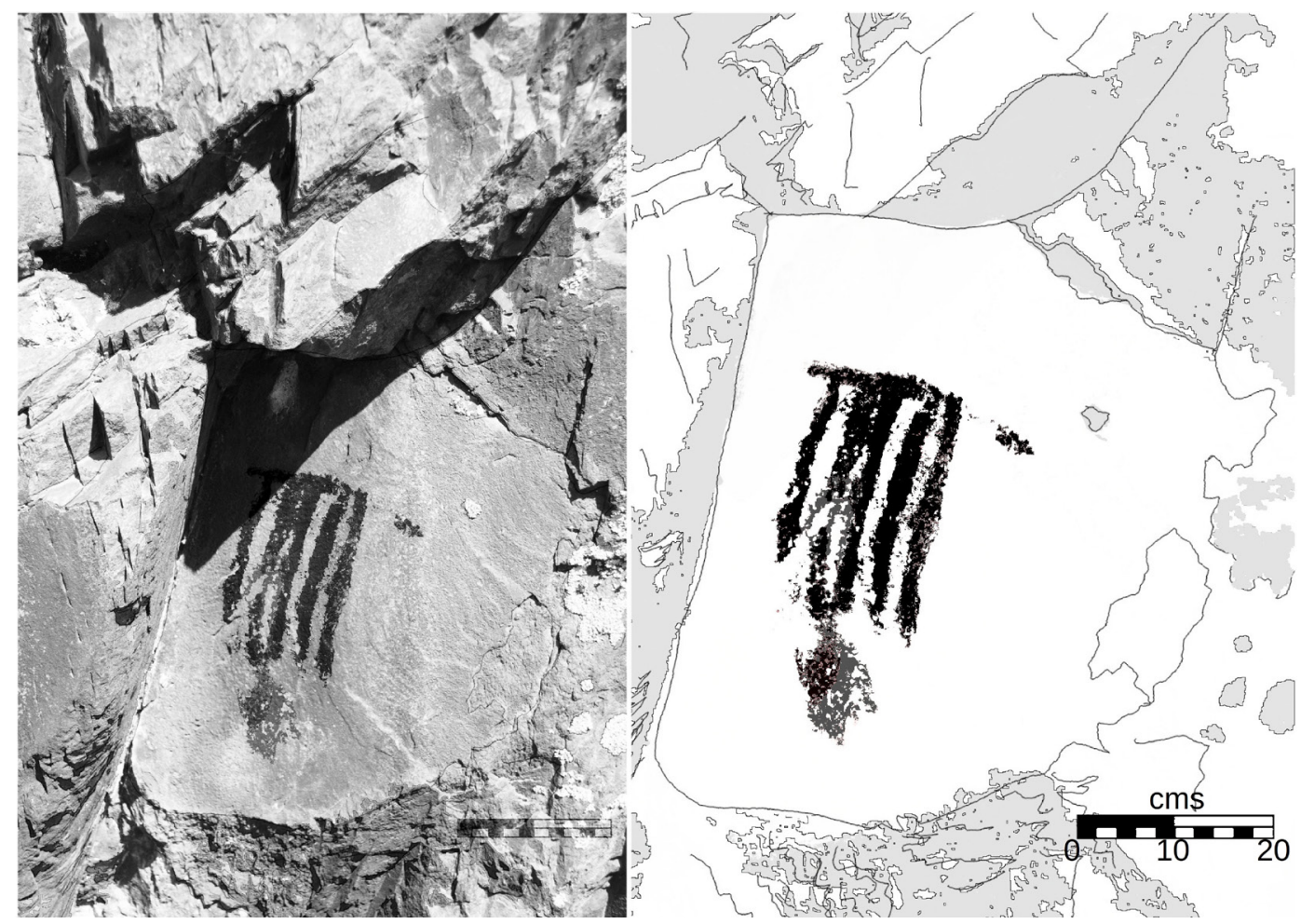

Fig. 4. Motivo 2 geométrico.

a estilos propuestos previamente (Gradín et al. 1976; Gradín, 1978).

En relación a los estilos definidos se desprendieron distintas visiones, especialmente en el área de la Patagonia meridional y específicamente en el sector del río Chico donde Felipe Bate (1970, 1971) identificó un subestilo denominado Río Chico asociado a motivos geométricos tales como puntos, trazos lineales y series paralelas, entre otros, preferentemente en rojo y negro y cuya datación relativa sería no posterior al nivel III de Cueva de Fell en la estratigrafía cultural de Junius Bird, por cuanto los niveles superiores cubren las pinturas más bajas de esta cueva (Bate, 1970:24). Destaca además la presencia de motivos conformados por figuras antropomorfas o "bailarines aislados". Además este subestilo sirvió de referente para los trabajos realizados en el sector de Cerro Benítez (Massone, 1982).

Posteriormente, y a partir de nuevas investigaciones llevadas a cabo en el sector (Gallardo, 2009; Sepúlveda, 2011), se logró establecer que las representaciones presentes en más de 20 sitios identificados, correspondían a motivos geométricos simples de formas regulares e irregulares, realizados a partir de trazos cortos y largos.

Aún cuando en los canales patagónicos los sitios con representaciones rupestres registrados son escasos (Fage, 2006; Mena et al. 2011; González et al. 2014), es posible señalar que las manifestaciones rupestres se pueden agrupar dentro del tipo de motivos geométricos simples y complejos, en donde predomina la utilización del color rojo, existiendo registro de otras tonalidades en menor medida, como es el caso del blanco para el sitio Alero Martín González Calderón (González et al. 2014), sitio en donde fue posible identificar, también, figuras antropomorfas y zoomorfas.

Dentro de este marco general y del estudio detallado de las representaciones presentes en el sitio Alero Picton 1 , se ha logrado determinar que el motivo 1, inicialmente definido como una línea vertical con cuatro trazados lineales, correspondería a la parte superior de una figura antropomorfa, cuya parte inferior no se encontraba representada posiblemente por el deplacamiento de un área del motivo, sumado al deterioro generado por agentes 
naturales. A través de programas especializados se logró identificar otro antropomorfo emplazado en el costado derecho del primero (Fig. 3).

Este tipo de motivo, como se mencionó anteriormente, se haya presente en Alero Martín González Calderón y en la Gruta del Pacífico en el archipiélago de Isla Madre de Dios, destacando en este último la utilización tanto de trazos lineales como sucesiones de puntos para formar la figura, técnica ausente en los dos sitios restantes. A su vez, no existe evidencia de esta clase de motivos para los sitios Cueva Mediana y Cueva Grande emplazados en los canales patagónicos septentrionales (Mena et al. 2011).

Por otro lado, se advierte que en los sitios registrados, incluidos Alero Picton 1, la coloración más utilizada corresponde al rojo, materia prima que en este caso pudo haber sido obtenida del mismo alero, debido a la presencia de vetas naturales de esta clase de pigmento, lo que ha sido postulado para otros estudios de sitios con pinturas rupestres de Patagonia (Sepúlveda, 2011; Muñoz \& Artigas, 2016). Sin embargo, para comprobar dicha hipótesis se hace necesaria la realización de estudios detallados en relación a este componente.

Por último, la asociación entre las pinturas y los conchales, donde se identifica principalmente material de tipo expeditivo para el caso de Alero Picton 1, también nos permite hacer un símil con el contexto presente en el alero Martín González Calderón (González et al. 2014).

\section{CONCLUSIÓN: PRIMEROS ALCANCES EN TORNO AL SITIO ALERO PICTON 1}

A partir de la información obtenida del registro preliminar de los motivos presentes en el sitio Alero Picton 1, se logra esbozar un panorama inicial de los yacimientos con este tipo de representaciones presentes en los canales australes.

En primera instancia y, a modo de hipótesis general sobre la base de la comparación con otros cuatro sitios registrados en la zona, se puede señalar que para este sector existe una mayor representatividad de la figura definida como antropoformo en comparación con el área continental, en donde se puede apreciar un aumento en los tipos de diseños, registrándose además motivos helioformes, zoomorfos equemáticos y posibles rastros de ñandú (Massone, 1982).

Por otro lado, el color rojo tiende a ser el color más utilizado, lo cual es extrapolable al resto de los sitios presentes en Patagonia meridional en donde, pese a observarse otras tonalidades tales como el negro o el blanco, siempre es el rojo la coloración más utilizada en la elaboración de motivos.

En cuanto a los grupos que realizaron este tipo de manifestaciones es posible reconocer que corresponden a poblaciones canoeras, ya que no existe otra forma de llegar a estos lugares sino es a través de una embarcación, además de evidenciarse conchales con material arqueológico en las inmediaciones adyacentes a los sitios. Probablemente, estos grupos son los mismos que se estarían asentando en las costas de la isla Navarino, e islas aledañas para el caso de Alero Picton 1, en donde se han registrado una serie de asentamientos datados entre 6.000 años AP hasta momentos históricos (Ortiz-Troncoso, 1972; Legoupil, 1993; Ocampo \& Rivas, 2000), quienes probablemente utilizaron la materia prima presente en el mismo alero para la realización de los motivos.

Finalmente, todas las hipótesis planteadas en este documento corresponden a aproximaciones iniciales basadas en la escasa información que se posee de esta clase de sitios y para el amplio territorio de los canales patagónicos. Por lo mismo se hace necesario la realización de nuevos estudios que involucren la excavación de los contextos asociados a los asentamientos con arte rupestre y el estudio detallado de los pigmentos que conforman los motivos, para de esta forma ir desentramando la complejidad que involucra este tipo de manifestaciones en el fin del mundo.

\section{BIBLIOGRAFÍA}

Artigas, D., \& Muñoz, C. (2015). Arte rupestre en el curso medio del río Ibáñez: retomando el camino de la interacción de las manifestaciones artísticas al contexto regional. En Actas del XIX Congreso Nacional de Arqueología Chilena, 507-514, Sociedad Chilena de Arqueología, Santiago.

Bate, F. (1970). Primeras investigaciones sobre el arte rupestre de la Patagonia chilena. Anales del Instituto de la Patagonia, 1, 17-25. 
Bate, F. (1971). Primeras investigaciones sobre el arte rupestre de la Patagonia chilena (segundo informe). Anales del Instituto de la Patagonia, 2, 33-41.

Carden, N. (2009). Imágenes a través del tiempo. Arte rupestre y construcción social del paisaje en la meseta central de Santa Cruz. Buenos Aires: Sociedad Argentina de Antropología.

Fage, L. H. (2006). Le mystère des indiens alakaluf. Ultima Patagonia, 14-21.

Gallardo, F. (2009). Sobre la composición y la disposición en el arte rupestre de Chile: consideraciones metodológicas e interpretativas. Magallania, 37(1), 85-98.

González, M., Gañán, M., \& Serrano, A. (2014). Primer registro de arte rupestre en Tierra del Fuego. Anales del Instituto de la Patagonia, 42(2), 175-181.

Gradín, C., Aschero, C., \& Aguerre, A. (1976). Investigaciones arqueológicas en la Cueva de las Manos estancia Alto Río Pinturas (provincia de Santa Cruz). Relaciones de la Sociedad Argentina de Antropología, 10, 201250.

Gradín, C. (1978). Algunos aspectos del análisis de las manifestaciones rupestres. Revista del Museo Provincial, 1, 120-137.

Harman, Jon. (2006). Decorrelation Stretch Enhancement of Great Mural Paintings of Baja California. En VI Encuentro Binacional: Balances y Perspectivas 2005 Frontera Dominica y Franciscana; La Alta y Baja California, Centro INAH, Baja California.

Legoupil, D. (1993). El archipiélago del Cabo de Hornos y la costa sur de la isla Navarino: poblamiento y modelos económicos. Anales del Instituto de la Patagonia, 22, 101-121

Lucero, V., \& Mena, F. (2000). Arte rupestre del río Ibáñez (XI región): un análisis cuantitativo exploratorio. En F. Morello, M. Martinic, A. Prieto \& G. Bahamonde (Ed.), Arqueología de fuego-patagonia. Levantando piedras, desenterrando huesos y develando arcanos (pp. 415-427). Punta Arenas: Ediciones CEQUA.

Massone, M. (1982). Nuevas investigaciones sobre el arte rupestre de Patagonia meridional chilena. Anales del Instituto de la Patagonia, 13, 73-94.

Mena, F., González, E., \& Labarca, R. (2011). Primeros registros de arte rupestre en el litoral de patagonia septentrional chilena. Magallania, 39(2), 303-307.

Muñoz, C., \& Artigas, D. (2016). Dar la mano y tomarse el todo: los sitios rupestres del Ibáñez medio como ventanas de un mundo abierto a los sistemas culturales amplios. En F. Mena (Ed.), Arqueología de la Patagonia: de mar a mar (pp. 58-66). Santiago: Ediciones CIEP.

Ocampo, C., \& Rivas, P. (2000). Nuevos fechados ${ }^{14} \mathrm{C}$ de la costa norte de la isla Navarino, costa sur del canal Beagle, provincia de Antártica Chilena, región de Magallanes. Anales del Instituto de la Patagonia, 28, 197-214.

Ortiz-Troncoso, O. (1972). Nota sobre un yacimiento arqueológico en el archipiélago del Cabo de Hornos. Anales del Instituto de la Patagonia, 3(1-2), 83-85.

Sepúlveda, M. (2011). Pinturas rupestres y tecnología del color en el extremo sur de Chile. Magallania, 39(1), 193210 . 
